\title{
Survival in patients treated for anaplastic meningioma
}

\author{
Jennifer Moliterno, MD, ${ }^{1}$ William P. Cope, BS, ${ }^{2}$ Emma D. Vartanian, BS, ${ }^{2}$ Anne S. Reiner, MPH, ${ }^{3}$ \\ Roselyn Kellen, BS, ${ }^{2}$ Shahiba Q. Ogilvie, MPH, ${ }^{2}$ Jason T. Huse, MD, PhD, ${ }^{4}$ and Philip H. Gutin, MD ${ }^{2}$
}

'Department of Neurosurgery, Yale University School of Medicine, New Haven, Connecticut; and Departments of ${ }^{2}$ Neurosurgery, ${ }^{3}$ Epidemiology and Biostatistics, and ${ }^{4}$ Pathology, Memorial Sloan-Kettering Cancer Center, New York, New York

\begin{abstract}
OBJECT While most meningiomas are benign, $1 \%-3 \%$ display anaplastic features, with little current understanding regarding the molecular mechanisms underlying their formation. In a large single-center cohort, the authors tested the hypothesis that two distinct subtypes of anaplastic meningiomas, those that arise de novo and those that progress from lower grade tumors, exist and exhibit different clinical behavior.

METHODS Pathology reports and clinical data of 37 patients treated between 1999 and 2012 for anaplastic meningioma at Memorial Sloan-Kettering Cancer Center (MSKCC) were retrospectively reviewed. Patients were divided into those whose tumors arose de novo and those whose tumors progressed from previously documented benign or atypical meningiomas.
\end{abstract}

RESULTS Overall, the median age at diagnosis was 59 years and $57 \%$ of patients were female. Most patients (38\%) underwent 2 craniotomies (range 1-5 surgeries) aimed at gross-total resection (GTR; 59\%), which afforded better survival when compared with subtotal resection according to Kaplan-Meier estimates (median overall survival [OS] 3.2 vs 1.3 years, respectively; $p=0.04$, log-rank test). Twenty-three patients $(62 \%)$ presented with apparently de novo anaplastic meningiomas. Compared with patients whose tumors had progressed from a lower grade, those patients with de novo tumors were significantly more likely to be female $(70 \%$ vs $36 \%$, respectively; $p=0.04)$, experience better survival (median OS 3.0 vs 2.4 years, respectively; $p=0.03$, log-rank test), and harbor cerebral hemispheric as opposed to skull base tumors ( $91 \%$ vs $43 \%$, respectively; $p=0.002$ ).

CONCLUSIONS Based on this single-center experience at MSKCC, anaplastic meningiomas, similar to glial tumors, can arise de novo or progress from lower grade tumors. These tumor groups appear to have distinct clinical behavior. De novo tumors may well be molecularly distinct, which is under further investigation. Aggressive GTR appears to confer an OS advantage in patients with anaplastic meningioma, and this is likely independent of tumor progression status. Similarly, those patients with de novo tumors experience a survival advantage likely independent of extent of resection.

http://thejns.org/doi/abs/10.3171/2014.10.JNS14502

KEY WORDS anaplastic meningioma; survival; outcome; oncology

A LTHOUGH meningiomas are the most common primary brain tumor, the anaplastic variant is exceedingly rare, accounting for $1 \%-3 \%$ of all meningiomas. ${ }^{16}$ These tumors display frankly anaplastic histology with an undeniably malignant clinical course. Median overall survival (OS) has been reported as 1.5 years,${ }^{28}$ with 5 -year survival ranging from $47 \%$ to $61 \%$.,11,30,32 Given the rarity of this meningioma variant and scarcity of literature, relatively little is understood with regards to the underlying pathogenesis and factors that impact clinical outcomes. While it has been well established that glioblastoma can present as either primary (i.e., arise de novo) or secondary (i.e., progress from a lower grade glioma), ${ }^{4,23,37}$ the notion of progression has been more obscure in meningioma, although it would appear that anaplastic meningiomas can similarly develop through both mechanisms..$^{6,12,38}$ Regardless of pathogenesis, treatment strategies have often consisted of aggressive resection followed by radiotherapy, with various chemotherapies implemented in end-stage disease as no standard medical management

ABBREVIATIONS CI = confidence interval; GTR = gross-total resection; MSKCC = Memorial Sloan-Kettering Cancer Center; OS = overall survival; STR = subtotal resection.

SUBMITTED March 12, 2014. ACCEPTED October 15, 2014.

INCLUDE WHEN CITING Published online April 10, 2015; DOI: 10.3171/2014.10.JNS14502.

DISCLOSURE The authors report no conflict of interest concerning the materials or methods used in this study or the findings specified in this paper. 
options currently exist. ${ }^{6,8,10,21,22,35}$ Traditionally it has been advocated that patients with anaplastic meningioma undergo aggressive gross-total resection (GTR). ${ }^{6,25,31,39}$ More recently, however, the impact of extent of resection has been questioned, suggesting that patients may do better after subtotal resection (STR) followed by adjuvant radiotherapy. ${ }^{32}$

Given the paucity of literature examining the existence and potential differences between de novo anaplastic meningioma and those that have progressed from a lower grade, and given the more recent questions regarding the impact of extent of resection on outcome, we reviewed our institutional experience with patients with anaplastic meningioma. We hypothesized that two distinct subtypes of anaplastic meningiomas exist and that they exhibit distinct clinical behaviors. Moreover, we sought to investigate those variables, such as extent of resection, which may impact OS.

\section{Methods \\ Data Collection}

The Departments of Neurosurgery and Pathology databases (Caisis and Copath, respectively) at Memorial Sloan-Kettering Cancer Center (MSKCC) were queried for all patients treated for anaplastic meningioma between 1999 and 2012 at our institution. The WHO 2000 classification criteria were used to render the histological diagnosis of anaplastic meningioma. ${ }^{13}$ The diagnosis of anaplastic meningioma in patients who were initially evaluated at another institution was re-reviewed and confirmed by the MSKCC Department of Pathology (J.T.H.). Patients' records, including reports from MSKCC and any previous information from referring institutions, were retrospectively reviewed. Information including demographics, pathology review, radiographic features, treatment history (such as number of resections, number and type of radiation treatments, and exposures to chemotherapy and brachytherapy), and status of systemic disease was compiled in an electronic database. Although there is no standard practice for treating patients with anaplastic meningiomas at MSKCC, these patients are more often than not offered upfront radiation therapy postoperatively following diagnosis. Decisions regarding type of radiation and number of times radiation is used at recurrence are often made on a case-by-case basis and commonly delivered at other hospitals. Mortality data were collected through death notifications in patient files when available, or via family contact.

\section{Tumor Classification}

With regards to progression status, patients were stratified into two subgroups, "de novo" and "progressed," based on a detailed review of all available pathology reports and clinical progress notes. A patient was considered to have a progressed anaplastic meningioma if there was prior documentation of a lower grade meningioma, either by histological diagnosis of a confirmed WHO Grade I or II tumor, and/or clinical documentation of a prior intracranial tumor in the same location as the current anaplastic meningioma. If no documentation or suspicion of prior diagnosis of meningioma existed, then the patient's tumor was considered de novo. The date of the first surgery revealing a histopathological diagnosis consistent with anaplastic meningioma was used to determine survival and progression-free intervals for patients with progressed tumors.

Magnetic resonance imaging studies were reviewed to assess tumor characteristics, as well as determine the anatomical location. Tumors were categorized as convexity (tumors overlying the lateral hemispheric convexities), parasagittal (midline along the falx), posterior fossa, or skull base (sphenoid wing, olfactory groove, or cerebellopontine angle). For statistical analysis purposes, parasagittal and posterior fossa tumors were combined with convexity and skull base, respectively.

Postoperative MR images, in conjunction with operative reports of the first surgical procedure for anaplastic meningioma, were reviewed to ascertain whether GTR or STR was achieved during the first surgery for anaplastic meningioma. These scans were performed within the initial postoperative period, i.e., within the 1st month of surgery. We defined GTR as complete removal of a tumor as measured by the surgeon's observation and/or evident on MRI. However, if postoperative imaging revealed any residual tumor, the surgery was considered an STR (Fig. 1).

\section{Statistical Analysis}

Overall survival was calculated from the date of the first anaplastic meningioma surgery to the date of death for those who died, or the date of the last follow-up evaluation for those who survived or were lost to follow-up. Patients who died were treated as events in all survival analyses and patients who survived or were lost to followup were censored in all survival analyses. Kaplan-Meier curves were used to visually depict survival and the logrank statistic was used to compare the univariate survival experiences of 2 or more groups. Cox proportional hazards models were used to examine variables and their univariate and multivariate association with OS. Although the sample size was small, it was of great a priori interest to investigate factors that were of univariate significance to determine if their association with OS was independent of other factors. Because sample size was small and number of events only allowed for multivariate modeling with two factors, we built two separate multivariate models to investigate location and resection with regard to OS, and progression status and resection with regard to OS. To examine associations between de novo/progressed tumors and other categorical characteristics, chi-square and Fisher's exact tests were used. To examine associations between de novo/progressed tumors and numeric variables, the Wilcoxon rank-sum test was used.

\section{Results}

Thirty-seven patients who met the aforementioned criteria and had complete medical records were included in this study. While the median follow-up duration was 4.04 years in the 9 patients alive at the time of completion of this study, the median follow-up duration in the entire cohort was 2.59 years. There was a slight female predomi- 


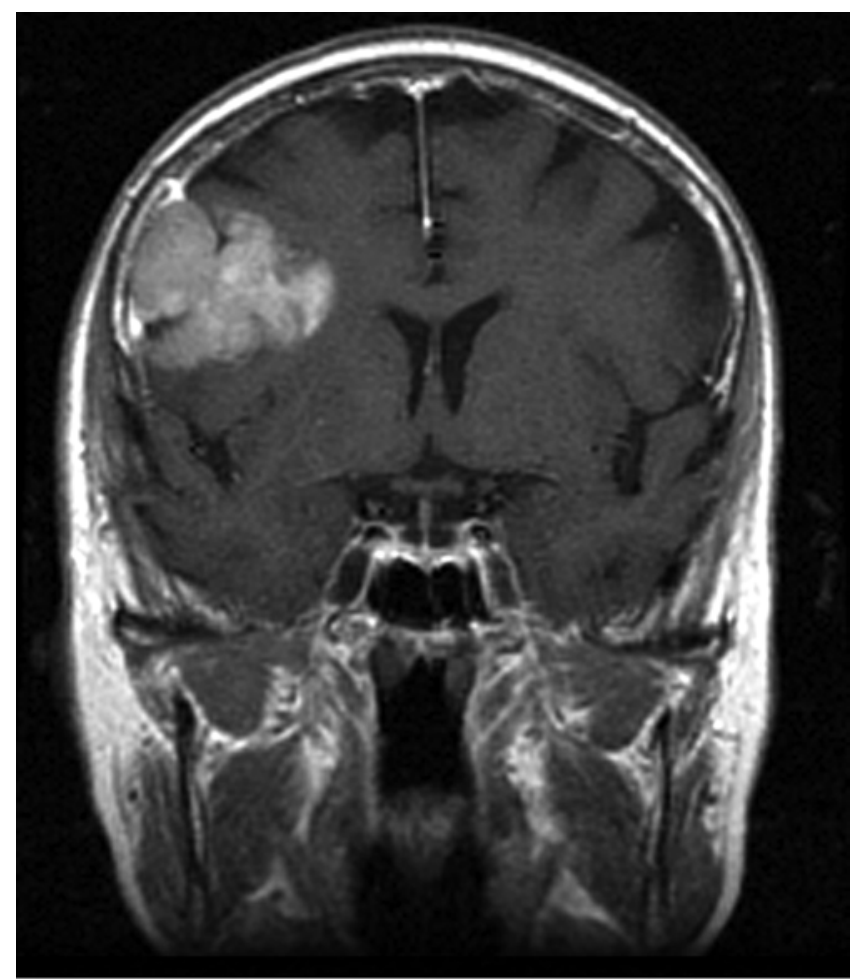

nance observed overall and the median age at diagnosis was 59 years (Table 1). The vast majority of patients $(\mathrm{n}=$ $27,73 \%$ ) had tumors located along the midline/parasagittal or cerebral convexities, and most patients underwent 2 craniotomies (38\%, range 1-5 surgeries) with GTR achieved in $59 \%$ of cases (Table 1).

\section{Radiotherapy and Chemotherapy}

Five patients $(14 \%)$ did not undergo radiotherapy. Of the 32 patients who did, most (38\%) were treated with two separate radiotherapy sessions (range $0-5$ radiotherapy applications), typically 1 initially and then 1 or more later for recurrence, and 6 patients (16\%) were treated with brachytherapy at recurrence. Most commonly, patients initially underwent conventional external beam radiation (typically with $5400 \mathrm{cGy}$ ), followed by single-dose stereotactic radiosurgery or stereotactic hypofractionated radiotherapy (1200-3000 cGy) as the subsequent radiation treatment, and type of radiotherapy was determined on a case-bycase basis factoring in the patient's functional status. Various chemotherapeutic agents were used in the end-stage

TABLE 1. Demographics and treatment characteristics for 37 patients with anaplastic meningioma

\begin{tabular}{|c|c|}
\hline Characteristics & No. of Patients (\%) \\
\hline No. of cases & 37 \\
\hline \multicolumn{2}{|l|}{ Sex } \\
\hline $\mathrm{F}$ & $21(57)$ \\
\hline M & $16(43)$ \\
\hline \multicolumn{2}{|l|}{ Age (yrs) } \\
\hline Median & 59 \\
\hline Range & $9-82$ \\
\hline \multicolumn{2}{|l|}{ Location } \\
\hline Convexity/parasagittal & $27(73)$ \\
\hline Skull base/posterior fossa & $10(27)$ \\
\hline \multicolumn{2}{|l|}{ Extent of resection* } \\
\hline GTR & $22(59)$ \\
\hline STR & $11(30)$ \\
\hline \multicolumn{2}{|l|}{ Total surgeries } \\
\hline 1 & $10(27)$ \\
\hline 2 & $14(38)$ \\
\hline 3 & $7(19)$ \\
\hline 4 & $4(11)$ \\
\hline 5 & $2(5)$ \\
\hline \multicolumn{2}{|l|}{ Total radiotherapies } \\
\hline 0 & $5(14)$ \\
\hline 1 & $10(27)$ \\
\hline 2 & $14(38)$ \\
\hline 3 & $7(19)$ \\
\hline 4 & $0(0)$ \\
\hline 5 & $1(3)$ \\
\hline \multicolumn{2}{|l|}{ Brachytherapy } \\
\hline Yes & $6(16)$ \\
\hline No & $31(84)$ \\
\hline
\end{tabular}

* Extent of resection was unable to be reliably determined in 4 patients. 
management of $38 \%$ of patients, and bevacizumab was the most common agent. Seven patients developed metastatic disease, and the lung and spine were the most frequent sites of disseminated anaplastic meningioma.

\section{Overall Survival}

Median OS was 2.7 years (Table 2). Two- and 5-year Kaplan-Meier estimates of OS were $66.6 \%$ (95\% confidence interval [CI] 51.1-82.0) and 27.9\% (95\% CI 12.543.2), respectively. There were 23 patients alive at 2 years and 7 patients alive at 5 years. Patients who underwent GTR during their first anaplastic meningioma surgery were found to have a significantly longer median OS (3.2 years) compared with patients who had undergone STR (1.3 years, $p=0.04$, log-rank test; Fig. 2A). Likewise, patients who harbored convexity or parasagittal anaplastic meningiomas lived significantly longer (3 years) than patients with tumors located along the skull base or posterior fossa (1.3 years; $p=0.02$, log-rank test; Fig. 2B). Brain invasion did not significantly affect median OS.

\section{Tumor Progression}

With regards to progression status, 23 patients (62\%) presented with apparent de novo anaplastic meningiomas. The remaining 14 patients (38\%) had evidence of having a tumor that progressed from a lower grade. Of the latter group, 6 patients' tumors progressed to anaplastic from benign (WHO Grade I), 6 patients progressed from atypical diagnosis (WHO Grade II), and 1 patient had documented progression from benign to atypical to anaplastic (the remaining patient had previous documentation of only "meningioma"). Using univariate analysis, de novo patients were found to have significantly longer median OS (3 years) than patients with progressed tumors (2.4 years; $\mathrm{p}=0.03$, log-rank test; Fig. $2 \mathrm{C}$ ). While there was no significant difference in age between the de novo and progressed patients, a female predominance was found among the former group $(n=16,70 \%$; Table 3$)$. Similarly, a significant difference in tumor location was found between the groups, such that de novo patients were more likely to have tumors located along the convexity/parasagittal location ( $p=0.002$; Table 3$)$. De novo tumors were far more likely to manifest with extracranial metastatic disease. Seven patients (18.9\%) harbored metastatic disease, all of whom had de novo tumors that were located along the convexity/parasagittal area. There was no significant difference in extent of resection between these groups. Finally, whether a patient with progressed tumor underwent radiation prior to anaplastic transformation was also not associated with survival.

\section{Variables Related to OS}

In multivariate analyses, we sought to determine whether progression status, tumor location, or extent of resection was independently driving the significant differences observed in survival. To achieve this goal with the sample size and number of death events in our study, we built two separate multivariate models as previously outlined. After adjusting for extent of resection, we found that patients who had first had lower grade tumors were twice
TABLE 2. Median OS in 37 patients with anaplastic meningioma

\begin{tabular}{lcc}
\hline \multicolumn{1}{c}{ Variable } & Median OS (yrs) & p Value \\
\hline Overall & 2.7 & - \\
\hline De novo vs progressed & & \\
\hline De novo & 3.0 & 0.03 \\
\hline Progressed & 2.4 & \\
\hline Resection & & \\
\hline GTR & 3.2 & 0.04 \\
\hline STR & 1.3 & \\
\hline Location & & \\
\hline Skull base/posterior fossa & 1.3 & 0.02 \\
\hline Convexity/parasagittal & 3.0 & \\
\hline Brain invasion & & \\
\hline No & 2.7 & 0.79 \\
\hline Yes & 2.8 & \\
\hline$*$ From logrank t. &
\end{tabular}

* From log-rank test.

as likely to die compared with de novo patients, and patients with convexity/parasagittal anaplastic meningiomas were $60 \%$ less likely to die compared with patients with tumors located along the skull base (Table 4). After adjusting for progression status, patients who underwent STR were found to be twice as likely to die compared with patients who underwent GTR (Table 4). Finally, after adjusting for location, patients who underwent STR were twice as likely to die as compared with patients who underwent GTR. These results all trended toward significance.

\section{Discussion}

Understanding the pathogenesis and clinical course of anaplastic meningiomas has long been challenging due to the rarity of the disease and small numbers of patients reported in the literature. In one of the largest single-center cohorts to date, we found that GTR, location, and subclassification of anaplastic meningioma as de novo rather than progressed confer a significant OS advantage. Median patient OS was found to be 2.7 years, approximately 1 year longer than previously reported in the literature. Two clinically distinct subgroups appear to exist based on progression status, and they display distinct clinical characteristics and behaviors. Patients with de novo anaplastic meningiomas are more likely to be female, harbor tumors located along the convexity/parasagittal region, and experience better OS compared with patients who develop anaplastic meningiomas via progression from lower grade tumors.

Gross-total resection specifically afforded a significantly better OS compared with patients who underwent STR. In multivariate analysis, we found that this trended toward significance and was likely independent of progression status. Surgical excision remains the primary modality of treating all grades of meningioma. ${ }^{10,21}$ However, a recent report by Sughrue et al. raised questions regarding the necessity of GTR in treating these highly malignant meningioma variants. ${ }^{32}$ In the largest single-center cohort to date, the authors demonstrated that STR yields slightly better OS than GTR. These results differ from the widely 

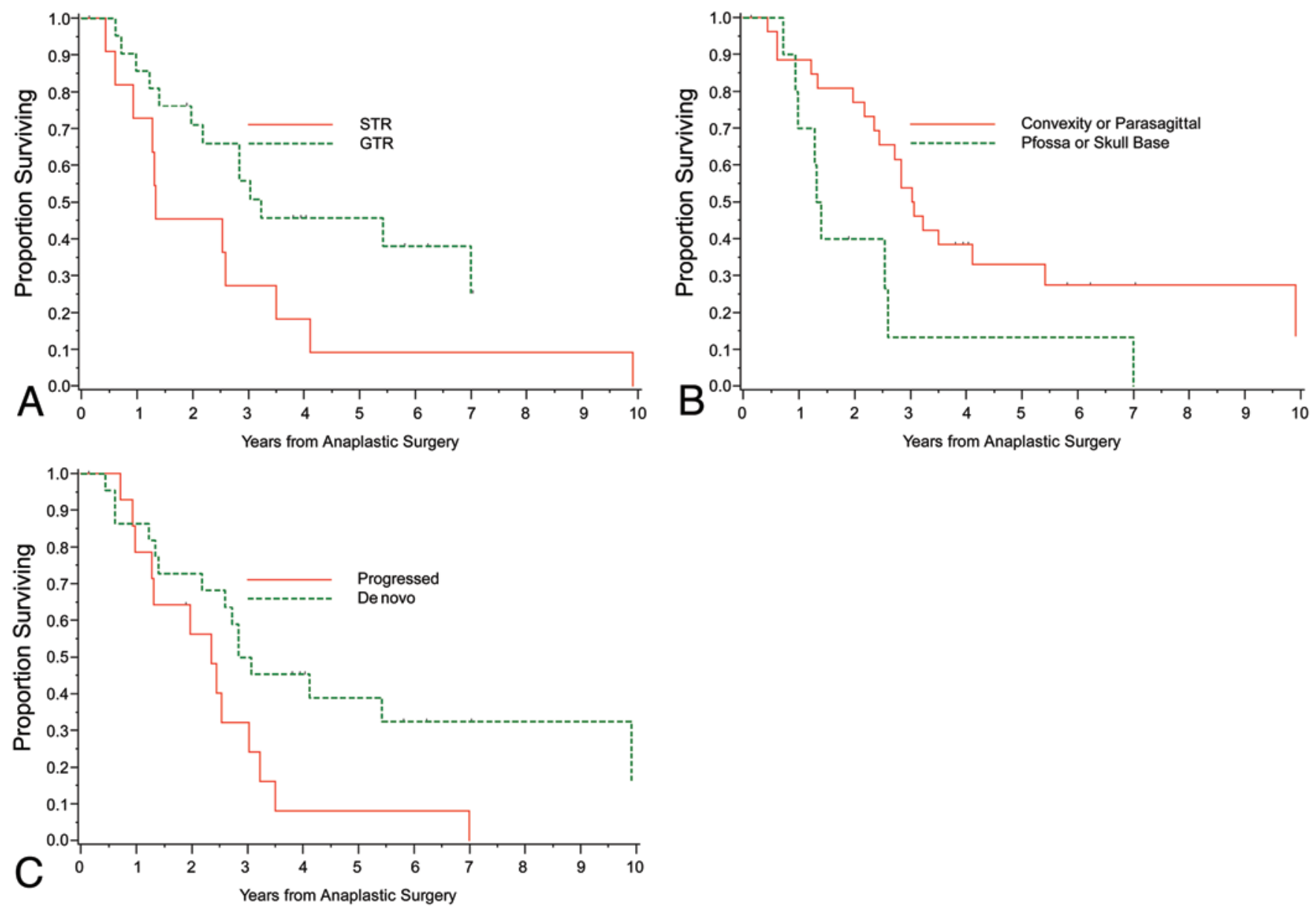

FIG. 2. Kaplan-Meier estimates of OS by according to resection status (A), tumor location (B), and progression status (C). Pfossa $=$ posterior fossa. Figure is available in color online only.

accepted management of brain tumors, as well as previous reports that have found that GTR is associated with better outcomes than STR in patients with anaplastic meningioma and that the extent of resection is a predictor of tumor recurrence. ${ }^{6,25,31,39}$ This information, coupled with our findings, would suggest that GTR should be considered the surgical goal for treating anaplastic meningiomas when it can be safely achieved.

A survival benefit associated with de novo tumors has been previously reported in the literature, but small sample sizes prohibited this finding from reaching statistical significance..$^{14}$ It has been postulated that this correlation could be driven by extent of resection such that surgery for de novo tumors may be technically easier compared with reoperations for progressed tumors. Such a surgical advantage could, in theory, render it easier to achieve GTR and perhaps aggressive resection may be responsible for improved survival in these patients. In our study, however, no difference was appreciated in patients with de novo versus progressed tumors with regards to extent of resection, suggesting both groups are similarly amenable to aggressive surgery. Moreover, the majority of patients with progressed tumors in our cohort underwent only 1 surgery prior to their anaplastic meningioma diagnosis, and thus we do not regard this as a significant confounder.
Although the statistical significance was lost in multivariate analysis due to power and sample size, we found that patients with de novo tumors likely experience a survival advantage independent of extent of resection. Likewise, aggressive GTR affords an OS advantage in patients with anaplastic meningioma, and this is also likely to be independent of progression status.

Others have found the convexity location of anaplastic meningiomas to be associated with significantly longer survival. ${ }^{25}$ Krayenbühl et al. ${ }^{14}$ combined data from multiple small studies of anaplastic meningioma to determine the distribution of locations of primary anaplastic meningioma tumors. Similar to the current study, the authors found that among 184 tumors, 73\% occurred at the convexity or parasagittal location. Our data display a similar distribution, although with a smaller proportion of parasagittal tumors. In comparing de novo and progressed cases, we found that the majority of progressed tumors were located along the skull base, while de novo cases were found almost exclusively in the convexities. Tumors arising in certain locations, such as at the cranial base, demonstrate significantly higher morbidity rates due to limitations on surgical excision and radiotherapy options. ${ }^{15,36}$ However, there were no significant differences between patients with de novo and progressed tumors in 
TABLE 3. Differences in characteristics and treatments by de novo versus progressed tumors

\begin{tabular}{lccc}
\hline \multicolumn{1}{c}{ Variable } & De Novo* & Progressed $^{*}$ & p Value† \\
\hline Sex & & & \\
\hline $\mathrm{M}$ & $7(30)$ & $9(64)$ & 0.04 \\
\hline $\mathrm{F}$ & $16(70)$ & $5(36)$ & \\
\hline Median total radiotherapies & 1 & 2 & 0.40 \\
\hline Total resection & & & \\
\hline GTR & $15(71)$ & $7(58)$ & 0.47 \\
\hline STR & $6(29)$ & $5(42)$ & \\
\hline Age & & & \\
\hline$\quad$ Median & 58 & 61 & 0.15 \\
\hline Location-new categories & & & \\
\hline$\quad$ Skull base/posterior fossa & $2(9)$ & $8(57)$ & 0.002 \\
\hline Convexity/parasagittal & $21(91)$ & $6(43)$ & \\
\hline
\end{tabular}

* All values given as number of patients (\%).

$\dagger$ Categorical variables compared with a chi-square or Fisher's exact test where appropriate. Numeric variables compared with a Wilcoxon rank-sum test.

regards to receipt of radiotherapy or GTR afforded in the present study.

While the female sex is a known risk factor associated with benign meningiomas, it has been reported that atypical and anaplastic variants are more common in males. ${ }^{11,18,26}$ Yet similar to the largest retrospective study of anaplastic meningiomas, ${ }^{32}$ we also found a slight female predominance. The majority of the patients in our cohort, however, had tumors that arose de novo, and we found such patients were more likely to be female. Although we cannot currently explain why women may be more likely to harbor de novo tumors, we speculate that this may explain the female predominance observed in the overall cohort.

The propensity for anaplastic meningiomas to metastasize extracranially has also been well documented in the literature, with reported incidences ranging from $7 \%$ to $43 \% .^{5,7,11,20}$ The lungs are the most common site of metastasis, ${ }^{5,7}$ but bone, liver, lymph node, and kidney sites have also been reported. ${ }^{9,17,19,33}$ Our reported incidence of $18.9 \%$ likely reflects the fact that our institution is a cancer referral center and commonly encounters patients with more extensive disease burden. Interestingly, all of the patients with metastatic disease harbored de novo tumors, all of which were located along the convexity/parasagittal location. A recent large-scale genetic analysis of sporadic nonNF2 mutant meningiomas revealed different underlying genetic driver mutations that correlated with intracranial location. ${ }^{3}$ Similarly, de novo and progressed anaplastic meningiomas may have different underlying driver mutations, which might explain the marked differences in intracranial location observed. While that study mainly focused on benign meningiomas, it underscores the importance of the molecular pathogenesis of these tumors and endorses the possibility that the genetic architecture of de novo and progressed anaplastic meningiomas may indeed be different and reflected in location and propensity for metastasizing.

The major limitation of this study lies within its retro-
TABLE 4. Multivariate survival analysis investigating tumor location, progression status, and extent of resection

\begin{tabular}{|c|c|c|c|}
\hline Level & $\mathrm{HR}^{*}$ & $95 \% \mathrm{Cl}$ & p Value \\
\hline \multicolumn{4}{|l|}{ Location } \\
\hline Skull base/posterior fossa & 1.00 & & \\
\hline Convexity/parasagittal & 0.41 & $0.17-1.01$ & 0.05 \\
\hline \multicolumn{4}{|l|}{ Resection } \\
\hline GTR & 1.00 & & \\
\hline STR & 2.02 & $0.88-4.66$ & 0.10 \\
\hline \multicolumn{4}{|l|}{ De novo vs progressed } \\
\hline De novo & 1.00 & & \\
\hline Progressed & 2.10 & $0.90-4.88$ & 0.09 \\
\hline \multicolumn{4}{|l|}{ Resection } \\
\hline GTR & 1.00 & & \\
\hline STR & 2.13 & $0.94-4.84$ & 0.07 \\
\hline
\end{tabular}

spective nature, most notably with the inability to definitively classify tumors as de novo versus progressed with complete accuracy. Although we have confidence in the completeness of the medical records of those patients included in the current study, there is truly no way to definitively know that a patient who initially presents with an anaplastic meningioma did not have a previous lower grade tumor that went undiagnosed for years. Thus, there is a possibility that a certain percentage of tumors deemed de novo are actually progressed tumors. Second, we elected to report on all patients with anaplastic meningioma who presented to our comprehensive cancer center, given the rarity of this disease. There is an inherent selection bias in this approach as we are unable to report on those patients with anaplastic meningiomas who did not choose to seek their care at MSKCC. Moreover, we chose to investigate OS rather than recurrence because many of our patients have been referred to our institution for part of their care, but receive follow-up care and radiation therapy elsewhere, rendering it difficult to evaluate for recurrence. Finally, although the current study is one of the largest in this area, the sample size was still relatively small. Although this was sufficient for univariate analyses, more complex modeling was underpowered.

Further investigation of potential differences in the underlying molecular basis of de novo and progressed anaplastic meningiomas, for instance, could better account for progression status and obviate the need to rely on clinical evidence alone. Genetic aberrations characteristic of anaplastic meningioma have been investigated, including losses on $6 \mathrm{q}, 10,14 \mathrm{q}, 9 \mathrm{p}$, and amplifications on $17 \mathrm{q} 23$, but progression status is almost never considered. ${ }^{24,29}$ One exception was found with Krayenbuhl et al., ${ }^{14}$ who specifically investigated several cytogenetic aberrations in de novo and progressed anaplastic meningiomas. Their data suggest a combination of cytogenetic changes, namely on chromosomes 1,14 , and 22 , is more often found in progressed rather than de novo tumors ( $71 \%$ vs $33 \%$, respectively), whereas monosomy of chromosomes 10 and 18 is more commonly found in progressed tumors compared 
with de novo ones $33 \%$ and $0 \%$ in progressed tumors, respectively, and $57 \%$ and $29 \%$ in de novo tumors, repsectively). ${ }^{14}$ Unfortunately, the small sample size (3 de novo and 7 transformed anaplastic meningiomas) precluded the authors from making any statistically significant conclusions. This work appears to be the only cytogenetic comparison of de novo and progressed anaplastic meningiomas, and no studies comparing the molecular genetics of these tumors exist to date.

Furthermore, controversy exists regarding the genesis of anaplastic meningioma. Classically, the formation of an anaplastic meningioma was believed to be a result of genetic changes on chromosome 22 , followed by a stepwise accumulation of other genetic alterations, ultimately resulting in a malignant tumor.,2,274 This notion was challenged by Al-Mefty et al., who demonstrated that 3 of 4 anaplastic meningioma specimens harbored complex genetic aberrations in the benign or atypical state prior to histopathological progression to malignancy, suggesting the lower grade tumors were predetermined to become anaplastic. ${ }^{1}$ While this study raises very interesting questions about the progression to malignant meningioma, it must be emphasized that only 4 patients' tumors were studied, and only a select few cytogenic aberrations were tested for. Ultimately, our understanding of anaplastic meningioma tumorigenesis may benefit from further insight into the underlying genetics of de novo and progressed anaplastic meningioma and may provide a powerful tool for better predicting clinical outcomes of this disease.

\section{Conclusions}

Anaplastic meningiomas appear to arise de novo or progress from lower grade tumors, and these groups have distinct clinical behavior. Aggressive GTR, de novo status, and location along the convexity/parasagittal intracranial areas are associated with better OS. Aggressive GTR affords a survival benefit likely independent of progression status, and similarly, those patients with de novo tumors likely experience a longer survival irrespective of extent of resection. Further studies focusing on the underlying molecular genetics of these tumors may provide a more useful way for predicting clinical behavior.

\section{References}

1. Al-Mefty O, Kadri PA, Pravdenkova S, Sawyer JR, Stangeby $\mathrm{C}$, Husain M: Malignant progression in meningioma: documentation of a series and analysis of cytogenetic findings. $\mathbf{J}$ Neurosurg 101:210-218, 2004

2. Boström J, Meyer-Puttlitz B, Wolter M, Blaschke B, Weber RG, Lichter P, et al: Alterations of the tumor suppressor genes CDKN2A (p16(INK4a)), p14(ARF), CDKN2B (p15(INK4b)), and CDKN2C (p18(INK4c)) in atypical and anaplastic meningiomas. Am J Pathol 159:661-669, 2001

3. Clark VE, Erson-Omay EZ, Serin A, Yin J, Cotney J, Ozduman K, et al: Genomic analysis of non-NF2 meningiomas reveals mutations in TRAF7, KLF4, AKT1, and SMO. Science 339: 1077-1080, 2013

4. Dropcho EJ, Soong SJ: The prognostic impact of prior low grade histology in patients with anaplastic gliomas: a casecontrol study. Neurology 47:684-690, 1996

5. Drummond KJ, Bittar RG, Fearnside MR: Metastatic atypical meningioma: case report and review of the literature. J Clin Neurosci 7:69-72, 2000
6. Dziuk TW, Woo S, Butler EB, Thornby J, Grossman R, Dennis WS, et al: Malignant meningioma: an indication for initial aggressive surgery and adjuvant radiotherapy. J Neurooncol 37:177-188, 1998

7. Enam SA, Abdulrauf S, Mehta B, Malik GM, Mahmood A: Metastasis in meningioma. Acta Neurochir (Wien) 138: 1172-1178, 1996

8. Engenhart-Cabillic R, Farhoud A, Sure U, Heinze S, Henzel M, Mennel HD, et al: Clinicopathologic features of aggressive meningioma emphasizing the role of radiotherapy in treatment. Strahlenther Onkol 182:641-646, 2006

9. Figueroa BE, Quint DJ, McKeever PE, Chandler WF: Extracranial metastatic meningioma. Br J Radiol 72:513-516, 1999

10. Hanft S, Canoll P, Bruce JN: A review of malignant meningiomas: diagnosis, characteristics, and treatment. J Neurooncol 99:433-443, 2010

11. Hug EB, Devries A, Thornton AF, Munzenride JE, Pardo FS, Hedley-Whyte ET, et al: Management of atypical and malignant meningiomas: role of high-dose, 3D-conformal radiation therapy. J Neurooncol 48:151-160, 2000

12. Jääskeläinen J, Haltia M, Servo A: Atypical and anaplastic meningiomas: radiology, surgery, radiotherapy, and outcome. Surg Neurol 25:233-242, 1986

13. Kleihues P, Sobin LH: World Health Organization classification of tumors. Cancer 88:2887, 2000

14. Krayenbühl N, Pravdenkova S, Al-Mefty O: De novo versus transformed atypical and anaplastic meningiomas: comparisons of clinical course, cytogenetics, cytokinetics, and outcome. Neurosurgery 61:495-504, 2007

15. Lamszus K: Meningioma pathology, genetics, and biology. J Neuropathol Exp Neurol 63:275-286, 2004

16. Louis DN, Ohgaki H, Wiestler OD, Cavenee WK, Burger PC, Jouvet A, et al: The 2007 WHO Classification of Tumours of the Central Nervous System. Acta Neuropathol 114:97-109, 2007 (Erratum in Acta Neuropathol 114:547, 2007)

17. Lüdemann WO, Obler R, Tatagiba M, Samii M: Seeding of malignant meningioma along a surgical trajectory on the scalp. Case report and review of the literature. J Neurosurg 97:683-686, 2002

18. Mahmood A, Caccamo DV, Tomecek FJ, Malik GM: Atypical and malignant meningiomas: a clinicopathological review. Neurosurgery 33:955-963, 1993

19. Miller DC, Ojemann RG, Proppe KH, McGinnis BD, Grillo HC: Benign metastasizing meningioma. Case report. J Neurosurg 62:763-766, 1985

20. Milosevic MF, Frost PJ, Laperriere NJ, Wong CS, Simpson WJ: Radiotherapy for atypical or malignant intracranial meningioma. Int J Radiat Oncol Biol Phys 34:817-822, 1996

21. Modha A, Gutin PH: Diagnosis and treatment of atypical and anaplastic meningiomas: a review. Neurosurgery 57:538550,2005

22. Nayak L, Iwamoto FM, Rudnick JD, Norden AD, Lee EQ, Drappatz J, et al: Atypical and anaplastic meningiomas treated with bevacizumab. J Neurooncol 109:187-193, 2012

23. Ohgaki H, Kleihues P: The definition of primary and secondary glioblastoma. Clin Cancer Res 19:764-772, 2013

24. Ozaki S, Nishizaki T, Ito H, Sasaki K: Comparative genomic hybridization analysis of genetic alterations associated with malignant progression of meningioma. J Neurooncol 41:167-174, 1999

25. Palma L, Celli P, Franco C, Cervoni L, Cantore G: Longterm prognosis for atypical and malignant meningiomas: a study of 71 surgical cases. J Neurosurg 86:793-800, 1997

26. Pasquier D, Bijmolt S, Veninga T, Rezvoy N, Villa S, Krengli $\mathrm{M}$, et al: Atypical and malignant meningioma: outcome and prognostic factors in 119 irradiated patients. A multicenter, retrospective study of the Rare Cancer Network. Int J Radiat Oncol Biol Phys 71:1388-1393, 2008 
27. Perry A, Banerjee R, Lohse CM, Kleinschmidt-DeMasters BK, Scheithauer BW: A role for chromosome 9p21 deletions in the malignant progression of meningiomas and the prognosis of anaplastic meningiomas. Brain Pathol 12:183-190, 2002

28. Perry A, Scheithauer BW, Stafford SL, Lohse CM, Wollan PC: "Malignancy" in meningiomas: a clinicopathologic study of 116 patients, with grading implications. Cancer 85:20462056, 1999

29. Riemenschneider MJ, Perry A, Reifenberger G: Histological classification and molecular genetics of meningiomas. Lancet Neurol 5:1045-1054, 2006

30. Rosenberg LA, Prayson RA, Lee J, Reddy C, Chao ST, Barnett GH, et al: Long-term experience with World Health Organization grade III (malignant) meningiomas at a single institution. Int J Radiat Oncol Biol Phys 74:427-432, 2009

31. Simpson D: The recurrence of intracranial meningiomas after surgical treatment. J Neurol Neurosurg Psychiatry 20:22-39, 1957

32. Sughrue ME, Sanai N, Shangari G, Parsa AT, Berger MS, McDermott MW: Outcome and survival following primary and repeat surgery for World Health Organization Grade III meningiomas. J Neurosurg 113:202-209, 2010

33. Tominaga T, Koshu K, Narita N, Yoshimoto T: Metastatic meningioma to the second cervical vertebral body: a case report. Neurosurgery 34:538-540, 1994

34. Weber RG, Boström J, Wolter M, Baudis M, Collins VP, Reifenberger G, et al: Analysis of genomic alterations in benign, atypical, and anaplastic meningiomas: toward a genetic model of meningioma progression. Proc Natl Acad Sci U S A 94:14719-14724, 1997

35. Wen PY, Quant E, Drappatz J, Beroukhim R, Norden AD: Medical therapies for meningiomas. J Neurooncol 99:365378,2010
36. Whittle IR, Smith C, Navoo P, Collie D: Meningiomas. Lancet $363: 1535-1543,2004$

37. Winger MJ, Macdonald DR, Cairncross JG: Supratentorial anaplastic gliomas in adults. The prognostic importance of extent of resection and prior low-grade glioma. J Neurosurg 71:487-493, 1989

38. Yamashita J, Handa H, Iwaki K, Abe M: Recurrence of intracranial meningiomas, with special reference to radiotherapy. Surg Neurol 14:33-40, 1980

39. Yang SY, Park CK, Park SH, Kim DG, Chung YS, Jung HW: Atypical and anaplastic meningiomas: prognostic implications of clinicopathological features. J Neurol Neurosurg Psychiatry 79:574-580, 2008

\section{Author Contributions}

Conception and design: Gutin, Moliterno, Cope, Ogilvie, Huse. Acquisition of data: Cope, Vartanian, Kellen, Ogilvie, Huse. Analysis and interpretation of data: Gutin, Moliterno, Cope, Vartanian, Ogilvie. Drafting the article: Moliterno, Cope, Reiner, Ogilvie. Critically revising the article: Gutin, Moliterno, Cope, Reiner, Ogilvie. Reviewed submitted version of manuscript: Gutin, Moliterno, Cope. Approved the final version of the manuscript on behalf of all authors: Gutin. Statistical analysis: Reiner. Administrative/technical/material support: Ogilvie. Study supervision: Gutin, Moliterno.

\section{Correspondence}

Philip H. Gutin, Department of Neurological Surgery, Memorial Sloan-Kettering Cancer Center, 1275 York Ave., C703, New York, NY 10028. email: gutinp@mskcc.org. 